\title{
ON THE SOLVABILITY OF A VARIATIONAL INEQUALITY PROBLEM AND APPLICATION TO A PROBLEM OF TWO MEMBRANES
}

\section{A. ADDOU and E. B. MERMRI}

(Received 17 March 2000)

\begin{abstract}
The purpose of this work is to give a continuous convex function, for which we can characterize the subdifferential, in order to reformulate a variational inequality problem: find $u=\left(u_{1}, u_{2}\right) \in K$ such that for all $v=\left(v_{1}, v_{2}\right) \in K, \int_{\Omega} \nabla u_{1} \nabla\left(v_{1}-u_{1}\right)+$ $\int_{\Omega} \nabla u_{2} \nabla\left(v_{2}-u_{2}\right)+(f, v-u) \geq 0$ as a system of independent equations, where $f$ belongs to $L^{2}(\Omega) \times L^{2}(\Omega)$ and $K=\left\{v \in H_{0}^{1}(\Omega) \times H_{0}^{1}(\Omega): v_{1} \geq v_{2}\right.$ a.e. in $\left.\Omega\right\}$.
\end{abstract}

2000 Mathematics Subject Classification. Primary 35J85.

1. Introduction. We are interested in the following variational inequality problem: find $u=\left(u_{1}, u_{2}\right) \in K$ such that for all $v=\left(v_{1}, v_{2}\right) \in K$,

$$
\int_{\Omega} \nabla u_{1} \nabla\left(v_{1}-u_{1}\right)+\int_{\Omega} \nabla u_{2} \nabla\left(v_{2}-u_{2}\right)+(f, v-u) \geq 0
$$

where $f$ belongs to $L^{2}(\Omega) \times L^{2}(\Omega)$ and $K$ is a closed convex set of $H_{0}^{1}(\Omega) \times H_{0}^{1}(\Omega)$ defined by

$$
K=\left\{v=\left(v_{1}, v_{2}\right) \in H_{0}^{1}(\Omega) \times H_{0}^{1}(\Omega): v_{1} \geq v_{2} \text { a.e. in } \Omega\right\} .
$$

Thanks to the orthogonal projection of the space $L^{2}(\Omega) \times L^{2}(\Omega)$ onto the cone $\mathscr{K}$ defined by

$$
\mathscr{K}=\left\{v=\left(v_{1}, v_{2}\right) \in L^{2}(\Omega) \times L^{2}(\Omega): v_{1} \geq v_{2} \text { a.e. in } \Omega\right\},
$$

we construct a functional $\varphi$ for which we can characterize the subdifferential at a point $u$, in order to reformulate problem (1.1) to a variational inequality without constraints; that is, find $u=\left(u_{1}, u_{2}\right) \in H_{0}^{1}(\Omega) \times H_{0}^{1}(\Omega)$ such that for all $v \in H_{0}^{1}(\Omega) \times H_{0}^{1}(\Omega)$,

$$
\int_{\Omega} \nabla u_{1} \nabla\left(v_{1}-u_{1}\right)+\int_{\Omega} \nabla u_{2} \nabla\left(v_{2}-u_{2}\right)+\varphi(v)-\varphi(u)+(h, v-u) \geq 0,
$$

where $\varphi$ is a continuous convex function from $H_{0}^{1}(\Omega) \times H_{0}^{1}(\Omega)$ to $\mathbb{R}$ and $h$ is an element of $L^{2}(\Omega) \times L^{2}(\Omega)$ depending only on $f$.

We prove that the solution $u=\left(u_{1}, u_{2}\right)$ can be obtained as a solution of a system of independent two Dirichlet problems

$$
u_{1}, u_{2} \in H_{0}^{1}(\Omega), \quad \Delta u_{1}=g_{1}, \quad \Delta u_{2}=g_{2} \text { in } \Omega,
$$

where $g_{1}$ and $g_{2}$ are two functions of $L^{2}(\Omega)$ determined in terms of $f_{1}$ and $f_{2}$. We will give an algorithm for computing these functions. 
This approach can be applied to study a variational inequality arising from a problem of two membranes [2].

2. Formulation of the problem. Let $\Omega$ be an open bounded set of $\mathbb{R}^{n}$ with smooth boundary $\partial \Omega$. We equip $H_{0}^{1}(\Omega) \times H_{0}^{1}(\Omega)$ with the norm

$$
a(u, v)=\int_{\Omega} \nabla u_{1} \nabla v_{1}+\int_{\Omega} \nabla u_{2} \nabla v_{2}
$$

where

$$
u=\left(u_{1}, u_{2}\right), v=\left(v_{1}, v_{2}\right) \in H_{0}^{1}(\Omega) \times H_{0}^{1}(\Omega) .
$$

For $r \in L^{2}(\Omega)$, we let

$$
r^{+}=\max \{r, 0\}, \quad r^{-}=\min \{r, 0\} .
$$

For $f=\left(f_{1}, f_{2}\right) \in L^{2}(\Omega) \times L^{2}(\Omega)$, we let

$$
f^{+}=\left(f_{1}^{+}, f_{2}^{-}\right), \quad f^{-}=\left(f_{1}^{-}, f_{2}^{+}\right) .
$$

For $v=\left(v_{1}, v_{2}\right) \in H_{0}^{1}(\Omega) \times H_{0}^{1}(\Omega)$, we let

$$
v_{+}=\left(v_{1}+\frac{\left(v_{2}-v_{1}\right)^{+}}{2}, v_{2}-\frac{\left(v_{2}-v_{1}\right)^{+}}{2}\right), \quad v_{-}=\left(-\frac{\left(v_{2}-v_{1}\right)^{+}}{2}, \frac{\left(v_{2}-v_{1}\right)^{+}}{2}\right)
$$

the projection of $v$ onto the cone $\mathscr{K}$ given by (1.3) with respect to the scalar product of $L^{2}(\Omega) \times L^{2}(\Omega)$ (respectively, the projection with respect to the scalar product of $L^{2}(\Omega) \times L^{2}(\Omega)$ on the polar cone of $\mathscr{K}$ defined by $\mathscr{K}^{0}=\left\{v=(-r, r) \in L^{2}(\Omega) \times L^{2}(\Omega)\right.$ : $r \geq 0$ a.e. on $\Omega\}$ ). We easily verify that

$$
a\left(v_{+}, v_{-}\right)=0
$$

for all $v \in H_{0}^{1}(\Omega) \times H_{0}^{1}(\Omega)$. A function $\varphi$ defined from $H_{0}^{1}(\Omega) \times H_{0}^{1}(\Omega)$ to $\mathbb{R}$ is called lower semi-continuous (l.s.c.) if its epigraph defined by

$$
\operatorname{epi}(\varphi)=\left\{v=\left(v_{1}, v_{2}\right) \in H_{0}^{1}(\Omega) \times H_{0}^{1}(\Omega), \lambda \in \mathbb{R}: \varphi(v) \leq \lambda\right\}
$$

is closed in $H_{0}^{1}(\Omega) \times H_{0}^{1}(\Omega) \times \mathbb{R}$. Let $u \in H_{0}^{1}(\Omega) \times H_{0}^{1}(\Omega)$, we denote by $\partial \varphi(u)$ the subdifferential of $\varphi$ at $u$, defined by

$$
\partial \varphi(u)=\left\{\mu \in H^{-1}(\Omega) \times H^{-1}(\Omega): \varphi(u)-\varphi(v) \leq\langle\mu, u-v\rangle \forall v \in H_{0}^{1}(\Omega) \times H_{0}^{1}(\Omega)\right\} .
$$

If $\varphi$ is a convex l.s.c. function, then for all $v \in H_{0}^{1}(\Omega) \times H_{0}^{1}(\Omega), \partial \varphi(v) \neq \varnothing$.

Let $f=\left(f_{1}, f_{2}\right) \in L^{2}(\Omega) \times L^{2}(\Omega)$. We denote by $(\cdot, \cdot)$ and $\|\cdot\|$ the scalar product and the norm of $L^{2}(\Omega) \times L^{2}(\Omega)$, respectively. We consider the following variational inequality problem: find $u=\left(u_{1}, u_{2}\right) \in K$ such that

$$
a(u, v-u)+(f, v-u) \geq 0 \quad \forall v=\left(v_{1}, v_{2}\right) \in K .
$$

It admits a unique solution. The functional $\varphi$ defined from $L^{2}(\Omega) \times L^{2}(\Omega)$ to $\mathbb{R}$ by $v \mapsto\left(f^{+}, v_{+}\right)$is continuous on $H_{0}^{1}(\Omega) \times H_{0}^{1}(\Omega)$ and convex. 
Proposition 2.1. $u=\left(u_{1}, u_{2}\right)$ is a solution of the problem (2.9) if and only if $u$ is the solution of the following problem: find $u=\left(u_{1}, u_{2}\right) \in H_{0}^{1}(\Omega) \times H_{0}^{1}(\Omega)$ such that

$$
a(u, v-u)+\varphi(v)-\varphi(u)+\left(f^{-}, v-u\right) \geq 0 \quad \forall v \in H_{0}^{1}(\Omega) \times H_{0}^{1}(\Omega) .
$$

Proof. It is well known in the general theory of variational inequalities that problem (2.10) admits a unique solution. So, it is sufficient to show that the solution $u$ of (2.10) is an element of $K$. Let $v=u_{+}$, then the inequality of (2.10) becomes

$$
a\left(u,-u_{-}\right)+\varphi(u)-\varphi(u)+\left(f^{-},-u_{-}\right) \geq 0 .
$$

By the relation (2.6) we deduce that $u_{-}=0$, hence $u \in K$.

Proposition 2.2. Problem (2.10) is equivalent to the following problem: find $\mu=$ $\left(\mu_{1}, \mu_{2}\right) \in L^{2}(\Omega) \times L^{2}(\Omega), u=\left(u_{1}, u_{2}\right) \in H_{0}^{1}(\Omega) \times H_{0}^{1}(\Omega)$,

$$
a(u, v)+(\mu, v)+\left(f^{-}, v\right)=0 \quad \forall v \in H_{0}^{1}(\Omega) \times H_{0}^{1}(\Omega), \mu \in \partial \varphi(u) .
$$

Proof. If $u \in H_{0}^{1}(\Omega) \times H_{0}^{1}(\Omega)$ and $\mu \in L^{2}(\Omega) \times L^{2}(\Omega)$ are the solution of (2.12), then by definition of $\mu \in \partial \varphi(u)$, we have

$$
a(u, v-u)+\varphi(v)-\varphi(u)+\left(f^{-}, v-u\right) \geq 0 \quad \forall v \in H_{0}^{1}(\Omega) \times H_{0}^{1}(\Omega) .
$$

Conversely, let $u$ be the solution of problem (2.10). For $v=u \pm w$, with $w \in H_{0}^{1}(\Omega) \times$ $H_{0}^{1}(\Omega)$, the inequality of (2.10) gives

$$
\begin{aligned}
& a(u, w)+\left(f^{-}, w\right) \geq-\left(f^{+}, w^{+}\right) \geq-\left\|f^{+}\right\|\|w\|, \\
& a(u, w)+\left(f^{-}, w\right) \leq\left(f^{+},(-w)^{+}\right) \leq\left\|f^{+}\right\|\|w\| .
\end{aligned}
$$

We deduce that

$$
\left|a(u, w)+\left(f^{-}, w\right)\right| \leq\left\|f^{+}\right\|\|w\| .
$$

So the linear form

$$
w \longmapsto a(u, w)+\left(f^{-}, w\right)
$$

is continuous on $H_{0}^{1}(\Omega) \times H_{0}^{1}(\Omega)$ equipped with the norm of $L^{2}(\Omega) \times L^{2}(\Omega)$. Where $\mu$ is an element of $L^{2}(\Omega) \times L^{2}(\Omega)$.

We set

$$
C=\left\{v \in L^{2}(\Omega) \times L^{2}(\Omega),(v, v) \leq \varphi(v) \forall v \in L^{2}(\Omega) \times L^{2}(\Omega)\right\} .
$$

LEMMA 2.3. Let $u \in L^{2}(\Omega) \times L^{2}(\Omega)$, then the following properties are equivalent:
(a) $\mu \in \partial \varphi(u)$.
(b) $\mu \in C$ and $(\mu, u)=\varphi(u)$.
(c) $\mu \in C$ and $(\nu-\mu, u) \leq 0$ for all $v \in C$.

Proof. (a) $\Rightarrow(\mathrm{b})$. Let $\mu \in \partial \varphi(u)$, we have

$$
\varphi(v)-\varphi(u) \geq(\mu, v-u) \quad \forall v \in L^{2}(\Omega) \times L^{2}(\Omega) .
$$


We put $v=0$, next $v=2 u$ in (2.18). Since $\varphi$ is positively homogeneous of degree 1 , we obtain $\varphi(u)=(\mu, u)$ and consequently

$$
\varphi(v) \geq(\mu, v) \quad \forall v \in L^{2}(\Omega) \times L^{2}(\Omega) .
$$

(c) $\Rightarrow$ (a). For all $v \in V$, we have

$$
(\mu, v-u) \leq \varphi(v)-(\mu, u) \leq \varphi(v)-(v, u) \quad \forall v \in C
$$

Hence for $v \in \partial \varphi(u)$, we have $(v, u)=\varphi(u)$, consequently $\mu \in \varphi(u)$.

We deduce from Lemma 2.3 the following relations:

$$
\mu_{1}+\mu_{2}=f_{1}^{+}+f_{2}^{-}, \quad f_{2}^{-} \leq \mu_{2} \leq \mu_{1} \leq f_{1}^{+} \text {a.e. in } \Omega \text {. }
$$

Indeed, the function $\varphi$ being positively homogeneous of degree $1, \mu \in \partial \varphi(u)$ implies

$$
\begin{aligned}
& (\mu, u)=\varphi(u), \\
& (\mu, v) \leq \varphi(v) \quad \forall v \in L^{2}(\Omega) \times L^{2}(\Omega) .
\end{aligned}
$$

Finally, it is sufficient to take in (2.23) elements $v=\left(v_{1}, v_{2}\right)$ with suitable choices on the components $v_{1}$ and $v_{2}$.

Let $V=H_{0}^{1}(\Omega) \times H_{0}^{1}(\Omega)$, and taking into account Lemma 2.3, we can write problem (2.12) as follows: find $u \in H_{0}^{1}(\Omega) \times H_{0}^{1}(\Omega), \mu \in C$,

$$
\begin{gathered}
a(u, v)+(\mu, v)+\left(f^{-}, v\right)=0 \quad \forall v \in H_{0}^{1}(\Omega) \times H_{0}^{1}(\Omega), \\
(v-\mu, u) \leq 0 \quad \forall v \in C .
\end{gathered}
$$

Let $A$ be the Riesz-Fréchet representation of $H^{-1}(\Omega) \times H^{-1}(\Omega)$ in $H_{0}^{1}(\Omega) \times H_{0}^{1}(\Omega)$. We set $M=A(C)$, this is a closed convex subset in $H_{0}^{1}(\Omega) \times H_{0}^{1}(\Omega)$ characterized by

$$
M=\left\{w \in H_{0}^{1}(\Omega) \times H_{0}^{1}(\Omega): a(w, v) \leq \varphi(v) \forall v \in H_{0}^{1}(\Omega) \times H_{0}^{1}(\Omega)\right\} .
$$

Problem (2.24) can be written in the following form: find $u \in H_{0}^{1}(\Omega) \times H_{0}^{1}(\Omega)$, $z \in M$,

$$
\begin{gathered}
a(u+z+t, v)=0 \quad \forall v \in H_{0}^{1}(\Omega) \times H_{0}^{1}(\Omega), \\
a(w-z, u) \leq 0 \quad \forall w \in M .
\end{gathered}
$$

with $z=A(\mu)$ and $t=A\left(f^{-}\right)$. Hence

$$
u=-z-t, \quad z=P_{M}(-t),
$$

where $P_{M}(-t)$ is the projection of $-t$ onto the closed convex set $M$ with respect to the scalar product $a(\cdot, \cdot)$ of $H_{0}^{1}(\Omega) \times H_{0}^{1}(\Omega)$.

From the equality of Proposition 2.2, we deduce that the solution $u$ of problem (2.9) verifies the following equations:

$$
\Delta u_{1}=\mu_{1}+f_{1}^{-}, \quad \Delta u_{2}=\mu_{2}+f_{2}^{+} \quad \text { in } \Omega .
$$


We notice that the prior knowledge of $\mu=\left(\mu_{1}, \mu_{2}\right)$ in terms of data of problem (2.9) yields the solutions $u_{1}$ and $u_{2}$ as solutions of two independent Dirichlet problems given by the system (2.28). We recall that for each element $f$ of $L^{p}(\Omega)$, the solution of the problem

$$
u \in H_{0}^{1}(\Omega), \quad-\Delta u=f \quad \text { in } \Omega,
$$

verifies the following properties (see [2]):

$$
u \in H^{2, p}(\Omega), \quad\|u\|_{H^{2, p}} \leq C\|f\|_{L^{p}},
$$

where $C$ is a constant depending only on $p$ and $\Omega$. We deduce from (2.28) that $u_{1}, u_{2}$ are in $H^{2}(\Omega)$ and

$$
\begin{aligned}
& \left\|u_{1}\right\|_{H^{2}(\Omega)} \leq c_{1}\left\|\mu_{1}+f_{1}^{-}\right\|_{L^{2}(\Omega)}, \\
& \left\|u_{2}\right\|_{H^{2}(\Omega)} \leq c_{2}\left\|\mu_{2}+f_{2}^{-}\right\|_{L^{2}(\Omega)}, \\
& \left\|u_{1}+u_{2}\right\|_{H^{2}(\Omega)} \leq c\left\|f_{1}+f_{2}\right\|_{L^{2}(\Omega)},
\end{aligned}
$$

where $c, c_{1}$, and $c_{2}$ are constants depending only on $\Omega$. We define the domain of noncoincidence [2] by

$$
\Omega^{+}=\left\{x \in \Omega: u_{1}(x)>u_{2}(x)\right\} .
$$

From relations (2.21), (2.22), and (2.23) we deduce that

$$
\mu_{1}=f_{1}^{+}, \quad \mu_{2}=f_{2}^{-} \quad \text { a.e. in } \Omega^{+} .
$$

When $u_{1}$ and $u_{2}$ are continuous on $\Omega$, the following relations are verified:

$$
\Delta u_{1}=f_{1}, \quad \Delta u_{2}=f_{2} \quad \text { in } \Omega^{+} .
$$

2.1. Algorithm for computing $z$. We consider the following projection problem:

$$
z \in H_{0}^{1}(\Omega) \times H_{0}^{1}(\Omega), \quad z=P_{M}\left(t^{\prime}\right), \quad \text { where } t^{\prime}=-t .
$$

Let $z_{0}$ belong to $M$, we compute the element $w_{0}$ of $M$ which verifies the following inequality:

$$
a\left(w-w_{0}, z_{0}-t^{\prime}\right) \geq 0 \quad \forall w \in M
$$

Next we compute

$$
z_{1}=P_{\left[z_{0}, w_{0}\right]}\left(t^{\prime}\right) .
$$

So, the algorithm is: $z_{n}$ being given in $M$, we construct $w_{n}$ verifying

$$
a\left(w-w_{n}, z_{n}-t^{\prime}\right) \geq 0 \quad \forall w \in M .
$$

Next $z_{n+1}=P_{\left[z_{n}, w_{n}\right]}\left(t^{\prime}\right)$. The sequence $\left\{z_{n}\right\}$ converges in $H_{0}^{1}(\Omega) \times H_{0}^{1}(\Omega)$ strongly to the solution of problem (2.35) [1]. Since $M=A(C)$, then the inequality (2.38) implies that there exists $\left\{v_{n}\right\}$ in $C$ which verifies

$$
\left(v-v_{n}, t^{\prime}-z_{n}\right) \leq 0 \quad \forall v \in C
$$

and Lemma 2.3 shows that $v_{n}$ is an element of $\partial \varphi\left(t^{\prime}-z_{n}\right)$. 
2.2. Application. This method of solvability can be applied to the study of a variational inequality arising from a problem of two membranes [2],

$$
\begin{aligned}
& \Delta u_{1}+\lambda u_{1}=f_{1}, \quad \Delta u_{2}=f_{2} \text { in } \Omega^{+}, \quad u_{1}=u_{2}, \\
& \frac{\partial u_{1}}{\partial x_{i}}=\frac{\partial u_{2}}{\partial x_{i}}, \quad 1 \leq i \leq n, \\
& \Delta u_{1}+\left(\frac{\lambda}{2}\right) u_{1}=\frac{1}{2}\left(f_{1}+f_{2}\right) \quad \text { in } \Omega^{-},
\end{aligned}
$$

where $\Omega^{+}$and $\Omega^{-}$, are two parts of $\Omega$ (unknown) separated by a hypersurface $\Gamma$ of $\mathbb{R}^{n}$ such that $\Omega=\Omega^{+} \cup \Gamma \cup \Omega^{-} ; f_{1}, f_{2}$ are two regular functions and $\lambda \in \mathbb{R}$. Formally, $\Omega^{+}$is the non-coincidence domain given by (2.32).

\section{REFERENCES}

[1] A. Degueil, Résolution par une méthode d'éléments finis d'un problème de Stephan en terme de temperature et en teneur en matériau non gelé, Thèse 3ème cycle, Université de Bordeaux, Bordeaux, 1977.

[2] D. Kinderlehrer and G. Stampacchia, An Introduction to Variational Inequalities and their Applications, Pure and Applied Mathematics, vol. 88, Academic Press [Harcourt Brace Jovanovich Publishers], New York, 1980. MR 81g:49013. Zbl 457.35001.

A. Addou: University Mohamed I, Faculty of Sciences, Department of Mathematics AND COMPUTER SCIENCES, OUJDA, MOROCCO

E-mail address: addou@sciences .univ-oujda.ac.ma

E. B. MeRmRi: UniVERSITY MOHAMEd I, FACUlTy OF SCIENCES, DePARTMENT OF MATHEMATICS AND COMPUTER SCIENCES, OUJDA, MOROCCO

E-mail address: mermri@sciences.univ-oujda.ac.ma, mermri@hotmai1.com 


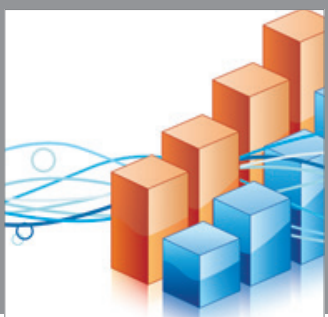

Advances in

Operations Research

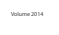

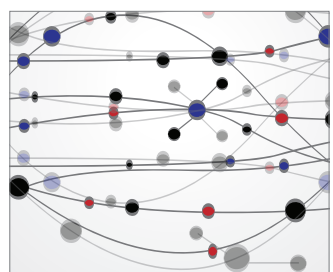

\section{The Scientific} World Journal
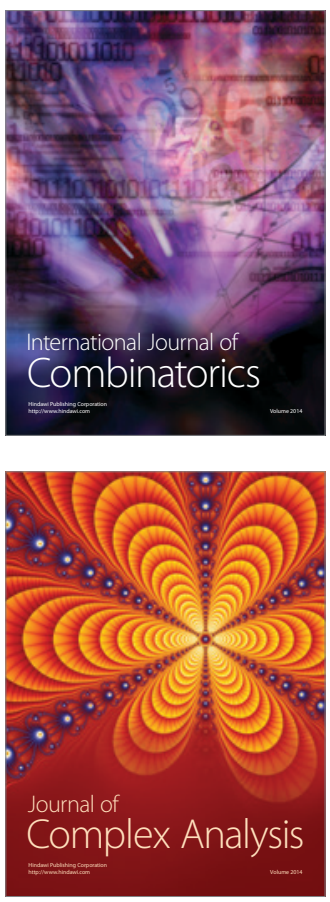

International Journal of

Mathematics and

Mathematical

Sciences
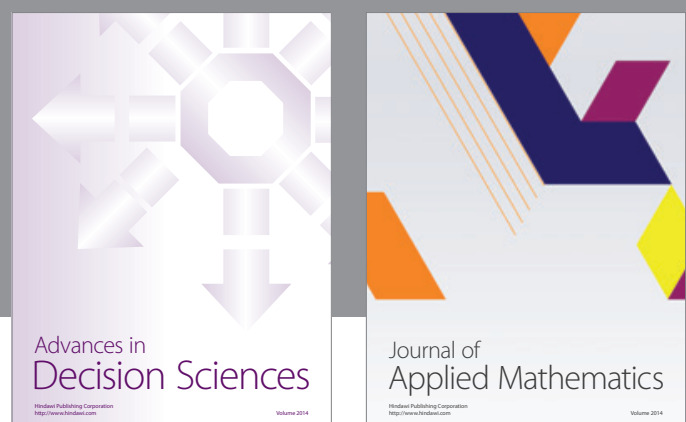

Journal of

Applied Mathematics
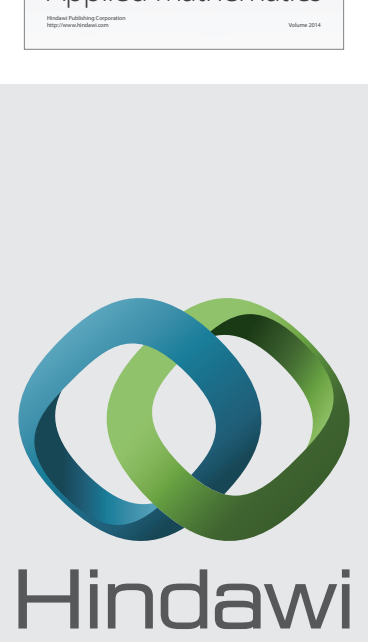

Submit your manuscripts at http://www.hindawi.com
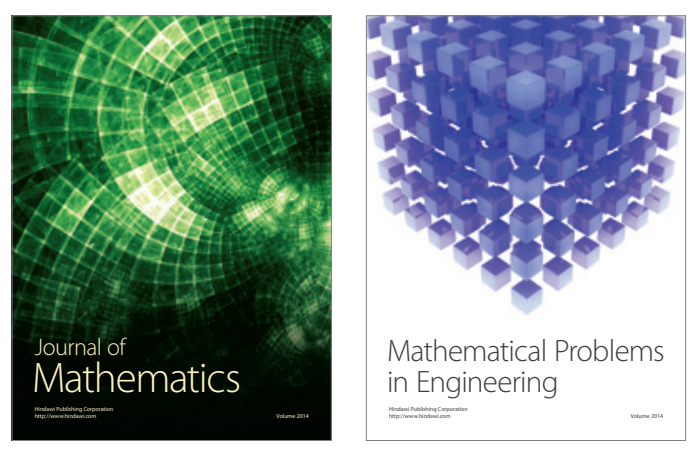

Mathematical Problems in Engineering
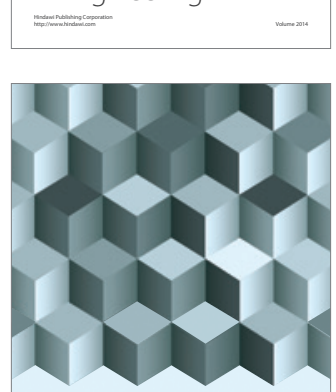

Journal of

Function Spaces
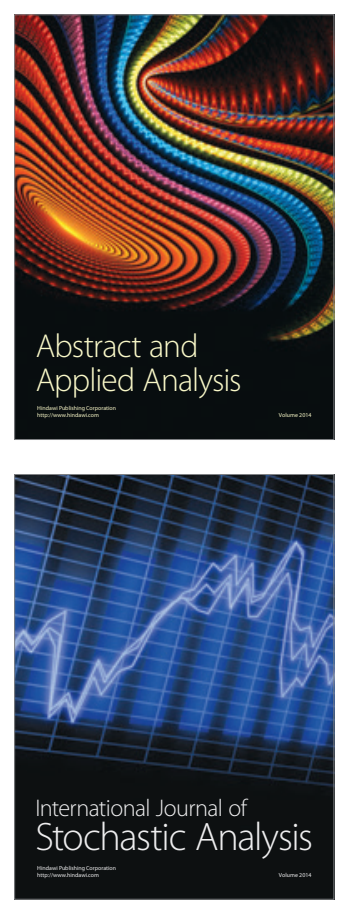

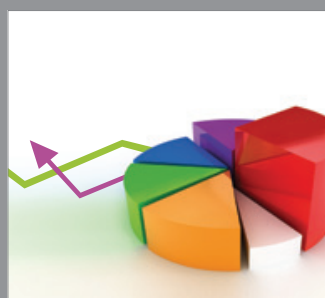

ournal of

Probability and Statistics

Promensencen
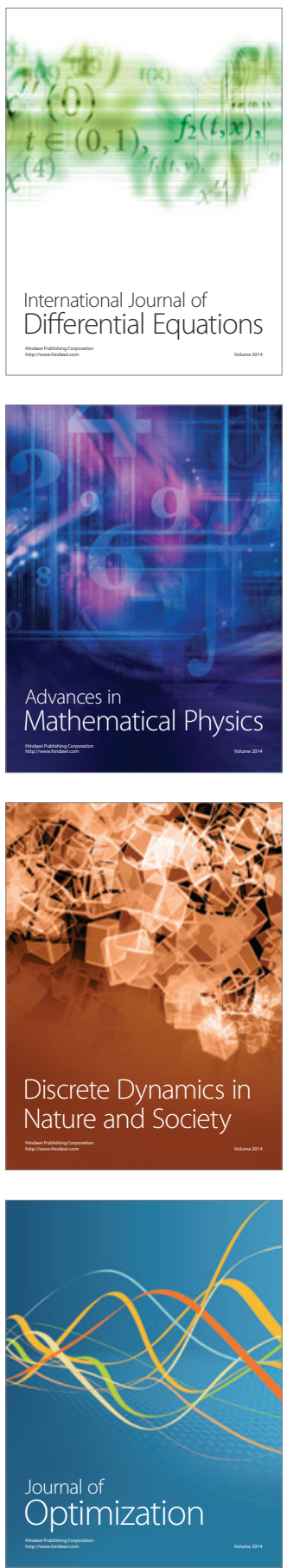